\title{
Easy Access to Antifungal Agents
}

Vulvovaginal candidiasis is a common problem; at one time or another most women experience at least one episode of this bothersome condition. For the past several years, the pharmaceutical industry has convinced women that this condition is easily treated and that they should recognize the symptoms of vulvovaginal candidiasis. Thus women have had access to a variety of intravaginal antifungal agents to treat this condition.

However, the symptoms can often be confusing; many physicians have difficulty in making a correct diagnosis. One also wonders why so many antifungal agents with a very similar spectrum of activity are available without a prescription. The effectiveness of these agents is similar and, in fact, not very efficacious. Yet women buy these products and spend millions of dollars without achieving suitable results. This is caused by one of three situations: the diagnosis is incorrect, the diagnosis is correct but the organism is not $C$. albicans and is likely to be resistant, or the organism is $C$. albicans but is not responsive to the agent.

The answer from the pharmaceutical industry and many of our colleagues is to develop a rapid test that can be performed by the patient. This may be suited for the individual who has an uncomplicated first episode and who does not have an associated condition-but this is a rare individual.

Why is this happening? Why is there a movement to encourage self-diagnosis? Perhaps physicians do not consider vaginitis a significant problem and do not learn or take the time to evaluate the vaginal ecosystem correctly. As a result they cannot make an accurate diagnosis leading to appropriate treatment.

Physicians must understand that the status of the vaginal ecosystem does affect the well being of both pregnant and nonpregnant patients. Even a "single" yeast infection in a pregnant patient can result in a significant problem, e.g., Candida chorioamnionitis.

Our concern should be to ensure that the treatment is specific and that an associated condition is not present that can place the patient in jeopardy of compromising her reproductive capabilities.

I believe that the infectious diseases societies must, once again, awaken our colleagues and raise the level of awareness regarding infectious diseases. We need to bring attention to all the conditions in the vaginal ecosystem that impact on women's health. This should be done not by rephrasing the same old song, but bringing our specialty into the twenty-first century by making our scientific data applicable to everyday clinical situations. Now is an opportune time for both societies to step forward, to be proactive and to make recommendations, and to work together to improve the health of women. 


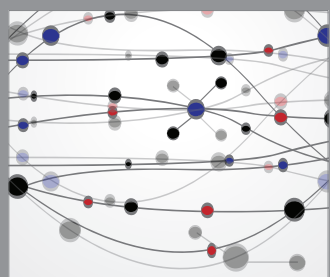

The Scientific World Journal
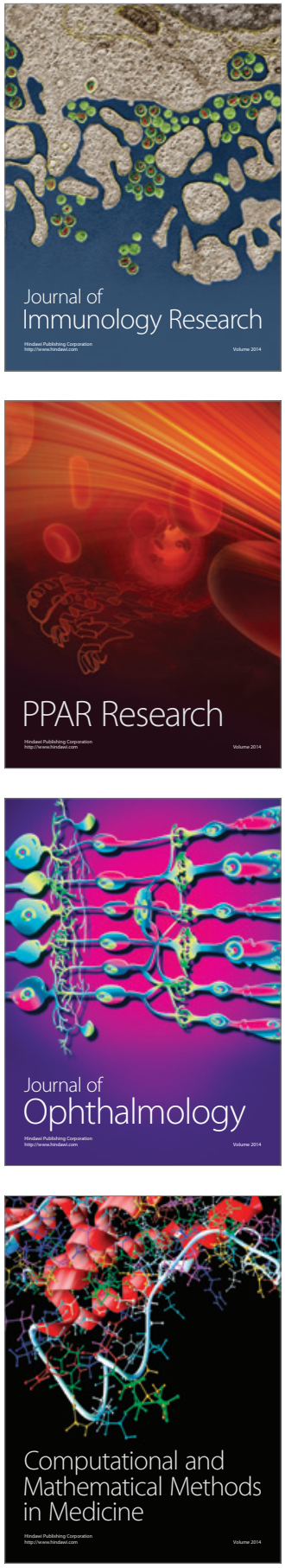

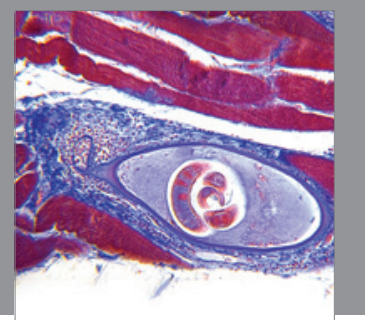

Gastroenterology

Research and Practice
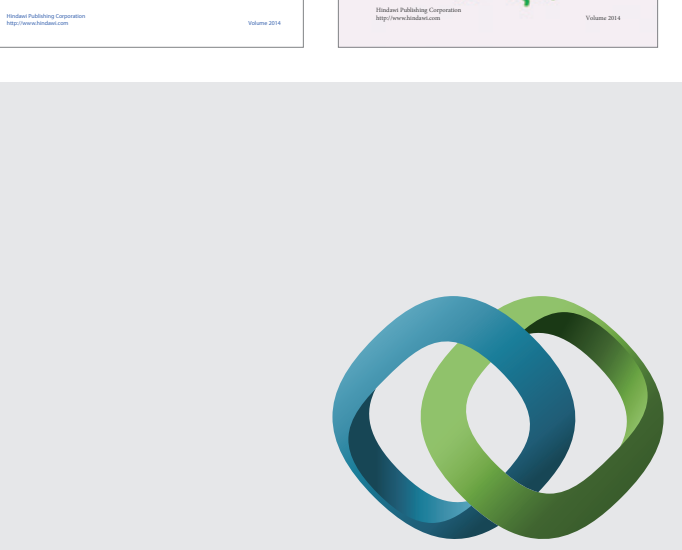

\section{Hindawi}

Submit your manuscripts at

http://www.hindawi.com
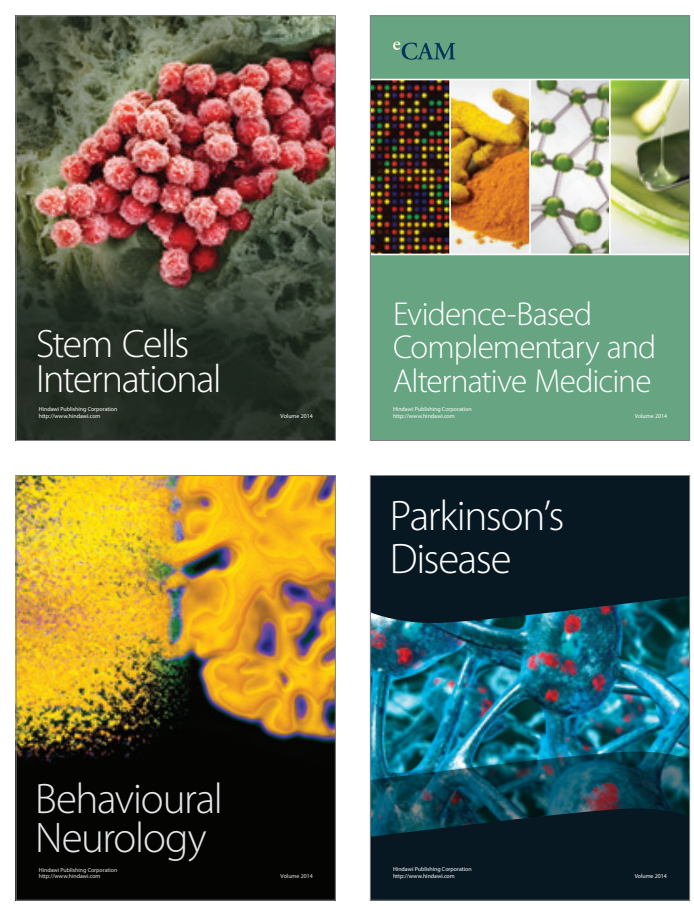

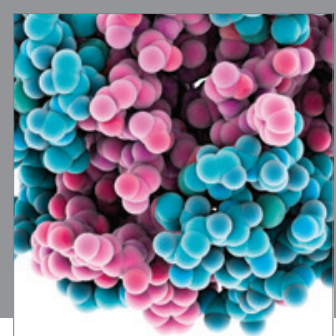

Journal of
Diabetes Research

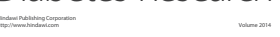

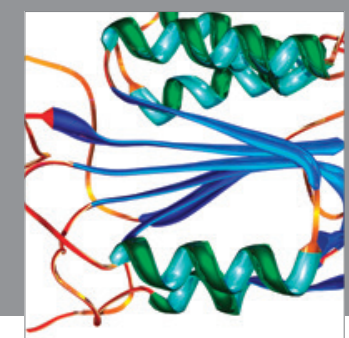

Disease Markers
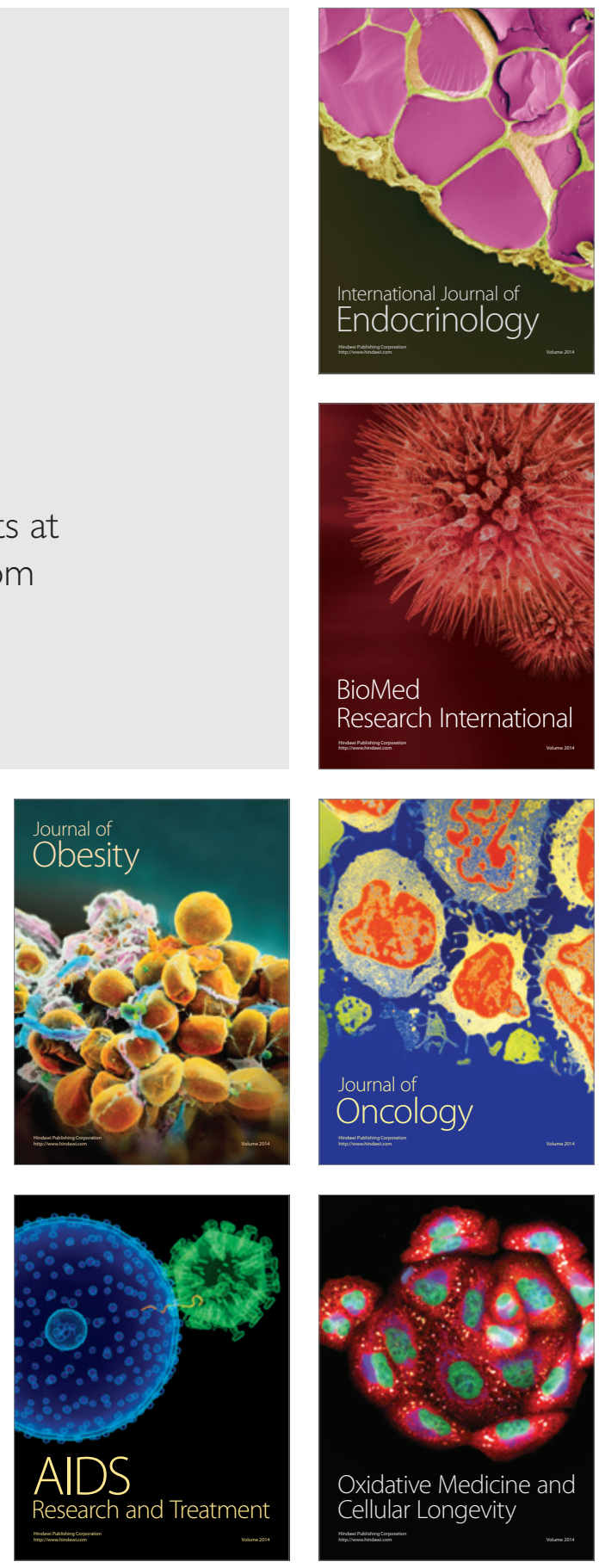